\title{
Application of Meta-heuristic Methods to Robust FACTS Controllers Design.
}

\author{
Lamia Kartobi ${ }^{1}$ and Mohamed Boudour ${ }^{2}$ \\ ${ }^{1}$ Department of Electrical Engineering \\ U.S.T.H.B. El Alia, BP.32, Bab Ezzouar, 16111, Algiers, ALGERIA. \\ e-mail: lamiakartobi@yahoo.fr \\ ${ }^{2}$ Department of Electrical Engineering \\ U.S.T.H.B. El Alia, BP.32, Bab Ezzouar, 16111, Algiers, ALGERIA. \\ e-mail:mboudour@ieee.org
}

\begin{abstract}
This paper presents a simple method to simultaneously tune Flexible AC Transmission Systems (FACTS) in multimachine power systems. The proposed approach employs Genetic Algorithms (GA) and particle Swarm Optimization (PSO). The objective is to maximise the minimum damping ratios, subject to all combinations of their locations. The proposed approach has been examined and tested on the 2-area, 4-machine system which is close to realistic interconnected power systems model. The results are promising and show the effectiveness and robustness of the proposed approach.
\end{abstract}

Keywords - Static Var Compensator, Genetic Algorithms, Particle Swarm Optimization, multimachine power systems.

\section{INTRODUCTION}

The increasing necessity of operating power systems near to their limits because of delays in building new transmission facilities and power plants and also because of the new regulation systems, are posing more and more interest in instability problems caused by low frequency inter-area oscillations. These oscillations occurring due to weak interconnected power systems are therefore becoming significant. Increasing the damping of these modes of oscillation by adequately tuning of Flexible AC Transmission Systems (FACTS); in particular the Static Var Compensator (SVC); had been the topic of many works. Many applications of the SVC have been suggested in the literature, such as, reduction of transmission line loss [1], the SVC has also received great attention in the damping of a power system [2], [3]. It can provide a supplementary damping torque for the synchronous generator and increase the damping of system oscillation. However, in the literature there are few papers with an implementation test report about the damping effect of an SVC [4], [3].

Genetic Algorithm (GA) is a powerful optimisation technique, independent on the complexity of problems where no prior knowledge is available. Many FACTS tuning methods using GA were presented in the literature. In [5], [6] GA had been applied for controllers design to power system stability enhancement. In [7] GA has been used to the damping of a system with a decentralized controller structure.

The particle Swarm Optimization (PSO) is an optimization algorithm where, one must have, at a given iteration a set of solutions or alternatives called particles (search agents) from one iteration to the following, each particle moves according to a rule that depends on: keeping records of the best point found by the particle in its past life and the current global best point found by the swarm of particles in their past life. The PSO sees its importance growing in the field of power systems. This method were applied and presented in several papers, such as, reactive power and voltage control in [8]. In [9], the PSO optimization were applied to control of microturbines in a power distribution system.

In this study, we propose a method to simultaneously tune SVCs in multimachine power systems using GA and PSO where the objective is to maximise the minimum damping ratios, subject to all combinations of their locations. The proposed tuning procedure is then applied to a 2-area 4machine system presented in [10] which is close to realistic interconnected power systems model. The robustness of the GA and PSO designed controllers is validated in tree operating conditions that represent considerable different scenarios including load variations and line outages.

\section{POWER SYSTEM MODELLING}

Thyristor controlled reactor with fixed capacitor (TCR + FC) is used as the SVC. Fig. 1 shows the schematic diagram of one phase of the three-phase SVC and its control block diagram [11], [12].

The primary function of the SVC is to control the reactive power and stabilise the system voltage. The auxiliary stabilising signal $U$ is added to the input of the SVC controller and fed into the excitation system to damp the machine oscillation.

The steady-state operating point of the SVC is given by [13]:

$$
I_{s}=B V_{t}
$$




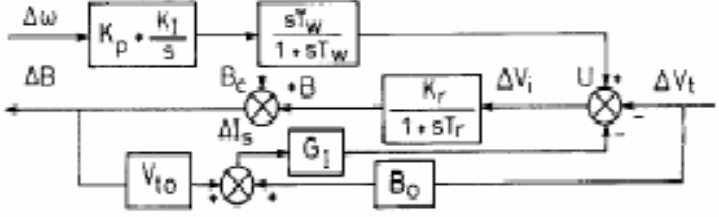

Fig. 1. SVC Model.

Linearising (1) about an operating point gives:

$$
\Delta I_{s}=B_{0} \Delta V_{t}+V_{t 0} \Delta B
$$

To derive a general relation corresponding to multimachine systems, assume that the SVC is located at the terminal bus of the synchronous generator. Furthermore, the input signal $\Delta V_{i}$ to the main control circuit of the SVC [Das 87] is given by:

$$
\Delta V_{i}=-\Delta V_{t}-G_{I} \Delta I_{s}+U
$$

A mathematical procedure of the proposed method corresponding to multimachine power system is derived. The system model is developed using the matrix approach including the SVC. Rearranging eqns. (2) and (3) gives:

$$
\begin{aligned}
& {\left[\Delta I_{s}\right]=\left[B_{0}\right]\left[A_{0}\right]\left[\Delta V_{t}\right]+\left[A_{0}\right]\left[V_{t 0}\right]\left[A_{0}\right]^{\mathrm{T}}[\Delta B]} \\
& {\left[\Delta V_{i}\right]=-\left[A_{0}\right]\left[\Delta V_{t}\right]-\left[A_{0}\right]\left[G_{I}\right]\left[A_{0}\right]^{\mathrm{T}}\left[\Delta I_{s}\right]+\left[A_{0}\right]\left[A_{0}\right]^{\mathrm{T}}[U]}
\end{aligned}
$$

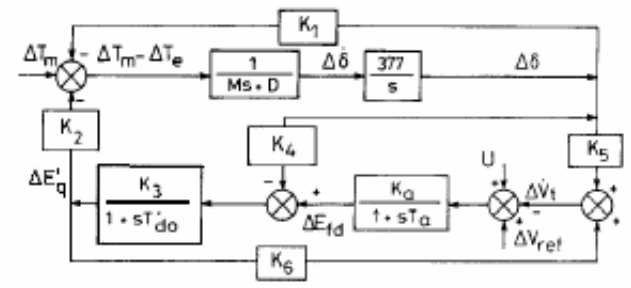

Fig. 2. linearised incremental model of synchronous machine with voltage regulator

From Figure 2, the incremental changes of voltage and electromagnetic torque are given by [15]:

$$
\begin{gathered}
{[\Delta V t]=\left[K_{5}\right][\Delta \delta]+\left[K_{6}\right]\left[\Delta E_{q}^{\prime}\right]} \\
{[\Delta T e]=\left[K_{1}\right][\Delta \delta]+\left[K_{2}\right]\left[\Delta E_{q}^{\prime}\right]}
\end{gathered}
$$

Also the steady-states field voltage $E_{f d}$ and the proportional voltage $E_{q}^{\prime}$ are given by:

$$
\begin{gathered}
{\left[\Delta E_{f d}\right]=-\left[K_{a}\right]\left[\Delta V_{t}\right]+\left[K_{a}\right]\left[A_{0}\right]^{\mathrm{T}}[U]+\left[K_{a}\right]\left[\Delta V_{r e f}\right]} \\
{\left[\Delta E_{q}^{\prime}\right]=\left[K_{3}\right]\left[\Delta E_{f d}\right]-\left[K_{3}\right]\left[K_{4}\right][\Delta \delta]}
\end{gathered}
$$

Substituting eqn. (9) into eqn. (6), gives

$$
\left[\Delta V_{t}\right]=\left[K_{5}-K_{6} K_{3} K_{4}\right][\Delta \delta]+\left[K_{6}\right]\left[K_{3}\right]\left[\Delta E_{f d}\right]
$$

Assuming $\Delta V_{\text {ref }}=0$, eqn. 4, 5, 8 and 10 give :
$\left[\Delta V_{t}\right]=\left[I+K_{c}\right]^{-1}\left[K_{d}\right][\Delta \delta]+\left[I+K_{c}\right]^{-1}\left[K_{c}\right]\left[A_{0}\right]^{\mathrm{T}}[U]$

Or

$$
\left[\Delta V_{t}\right]=\left[H_{Q}\right][\Delta \delta]+\left[H_{D}\right][U]
$$

And

$\left[\Delta V_{i}\right]=-\left[A_{0}+A_{0} G_{I} A_{0}{ }^{T} B_{0} A_{0}\right]\left[H_{Q}\right][\Delta \delta]-$

$\left[A_{0}\right]\left[G_{I}\right]\left[A_{0}\right]^{\mathrm{T}}\left[A_{0}\right]\left[V_{t 0}\right]\left[A_{0}\right]^{\mathrm{T}}[\Delta B]+$

$\left[A_{0} A_{0}{ }^{T}-\left(A_{0}+A_{0} G_{I} A_{0}{ }^{T} B_{0} A_{0}\right) H_{D}\right][U]$

The steady-state change of the SVC suceptance is given by:

$$
[\Delta B]=\left[K_{r}\right]\left[\Delta V_{i}\right]
$$

From eqn. 12, 13 and 14, we obtain:

$$
[\Delta B]=-\left[K_{8}\right][\Delta \delta]+\left[K_{9}\right][U]
$$

Rearranging eqn. 15 gives:

$$
[U]=\left[K_{9}\right]^{-1}[\Delta B]+\left[K_{9}\right]^{-1}\left[K_{8}\right][\Delta \delta]
$$

Eqns. 8, 9 and 16 give

$$
\left[\Delta E_{q}^{\prime}\right]=\left[K_{10}\right][\Delta \delta]+\left[K_{11}\right][\Delta B]
$$

Also, eqns. $17,7,16$ and 12 give

$$
\begin{aligned}
& {\left[\Delta T_{e}\right]=\left[K_{1}+K_{2} K_{10}\right][\Delta \delta]+\left[K_{2}\right]\left[K_{11}\right][\Delta B]} \\
& {\left[\Delta V_{t}\right]=\left[H_{Q}+H_{D} K_{9}^{-1} K_{8}\right][\Delta \delta]+\left[H_{D}\right]\left[K_{9}\right]^{-1}[\Delta B]}
\end{aligned}
$$
or:

$$
\begin{gathered}
{\left[\Delta T_{e}\right]=\left[K_{12}\right][\Delta \delta]+\left[K_{13}\right][\Delta B]} \\
{\left[\Delta V_{t}\right]=[C][\Delta \delta]+[W][\Delta B]}
\end{gathered}
$$

where:

$$
\begin{aligned}
& {\left[K_{d}\right]=\left[K_{5}\right]-\left[K_{6}\right]\left[K_{3}\right]\left[K_{4}\right],\left[K_{c}\right]=\left[K_{6}\right]\left[K_{3}\right]\left[K_{a}\right]} \\
& {\left[K_{8}\right]=\left[I+K_{r} A_{0} G_{I} A_{0}^{T} A_{0} V_{t 0} A_{0}^{T}\right]^{-1}\left[K_{h}\right]\left[H_{Q}\right]} \\
& {\left[K_{\theta}\right]=\left[I+K_{r} A_{0} G_{I} A_{0}^{T} A_{0} V_{t 0} A_{0}^{T}\right]^{-1}\left[K_{r} A_{0} A_{0}^{T}-K_{h} H_{D}\right]} \\
& {\left[K_{10}\right]=\left[K_{3} K_{a}\left(A_{0}^{T}-H_{D}\right) K_{9}^{-1} K_{8}-\left(K_{3} K_{a} H_{Q}+K_{3} K_{4}\right)\right]} \\
& {\left[K_{h}\right]=\left[K_{r}\right]\left[A_{0}+A_{0} G_{I} A_{0}^{T} B_{0} A_{0}\right],[W]=\left[H_{D}\right]\left[K_{9}\right]^{-1}} \\
& {\left[K_{11}\right]=\left[K_{3} K_{a}\left(A_{0}^{T}-H_{D}\right)\right]\left[K_{9}\right]^{-1}, K_{13}=K_{2} K_{11}} \\
& {\left[K_{12}\right]=\left[K_{1}+K_{2} K_{10}\right],[C]=\left[H_{Q}+H_{D} K_{9}^{-1} K_{8}\right]} \\
& H_{Q}=\left[I+K_{c}\right]^{-1}\left[K_{d}\right], \text { and }\left[H_{D}\right]=\left[I+K_{c}\right]^{-1}\left[K_{c}\right]\left[A_{0}\right]^{T}
\end{aligned}
$$

The parameters, $K_{1}-K_{6}, K_{d}, K_{c}, K_{10}, K_{w} C, K_{12}, G_{l}$, and $H_{Q}$ form square matrices each of order $(n g \times n g)$ and $H_{D}, W, K_{11}$ and $K_{13}$ are matrices of order $(n g \times n s v c)$. Also, $K_{8}$ and $A_{0}$ are matrices of order (nsvc $\times n g$ ) and $K_{r}, B_{0}$ and $K_{9}$ form square matrices each of order ( $n s v c$ $\times n s v c$ ), where $T$ is the transpose of the matrix, and $A_{0}$ is given by

$$
\begin{aligned}
& 1 \quad \cdots \quad \cdots \quad n s v c \quad n g \\
& A_{0}=\begin{array}{l}
1 \\
\vdots \\
\vdots \text { nsvc }
\end{array}\left(\begin{array}{ccccccc}
1 & 0 & 0 & \ldots & \ldots & \ldots & 0 \\
0 & 1 & 0 & \ldots & \ldots & \ldots & 0 \\
0 & 0 & 1 & 0 & \ldots & \ldots & 0 \\
0 & 0 & 0 & 1 & 0 & \cdots & 0
\end{array}\right)
\end{aligned}
$$


If $n s v c=n g$ (i.e. SVC located at all generator terminals) the $A_{0}$. matrix is unity diagonal matrix, and if $A_{0}=0$ (i.e. no SVC located at generator terminals).

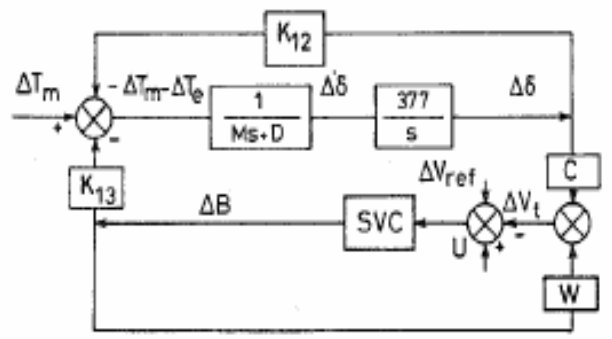

Fig. 3 Synchronous machine model with new controller parameters.

Eqn. (20) represents a new formula of the developed electromagnetic torque. The first term represents the synchronising torque coefficient, which must be positive to ensure system stability. The second term represents the degree to which a change in SVC susceptance can cause a relative acceleration of the machines. This term may be defined as the effectiveness factor of the SVC. Raising this factor gives more system stability.

Fig. 3 shows the final basic model of machine containing the effect of SVC compensation.

In the following studies, the output signals $\Delta w, U$ are fed back to the corresponding input of the SVC controller gain [16], and incorporated in each machine, shown in Fig. 3, which can be modelled by an equivalent second-order differential equation. The state equation of the system can be expressed as follows:

$$
\dot{X}=A X+F \Delta B
$$

where $X$ is the state vector and $\Delta B$ is the control signal.

\section{EVOLUTIONARY ALGORITHMS BASICS}

\section{A. Genetic Algorithm}

Genetic Algorithms are search procedures inspired from the mechanisms of evolution and natural genetics. They combine the survival of the fittest principle with information exchange among individuals to produce computationally simple yet powerful tools for system optimization and other applications [17].

The first step in the solution of an optimization problem using GA is the encoding of the optimization problem's variables often referred to as chromosomes by analogy to the natural genetic process. Secondly, the objective function is converted to a fitness function which normalizes the objective function to a convenient range and, then, is used to evaluate each string. An initial population of solution alternatives is usually chosen at random. Potentially good candidate solutions known beforehand can be included in the initial population in order to speed up computation and increase the chances of finding the global optimum.
Selection is responsible for the implementation of the survival of the fittest principle. Crossover implements information exchange among individuals of a population in an attempt to generate better fitted new individuals. Mutation has the role of restoring good genetic material that may have been lost by selection and crossover. There are different ways of performing selection, crossover, and mutation. The values of $p_{c}$ and $p_{m}$ and the population size are referred to as the control parameters of the GA. Their choice determines the speed of convergence and the probability of finding the optimum solution. Typical values for these parameters are [18]: population size of 30 to 200, crossover rates from 0.5 to 1.0 , and mutation rates from 0.001 to 0.05 .

\section{B. Particle Swarm Optimisation}

Natural creatures sometimes behave as a swarm. One of the main streams of artificial life research is to examine how natural creatures behave as a swarm and reconfigure the swarm models inside a computer. Swarm behavior can be modeled with a few simple rules. A school of fish and a swarm of birds can be modeled with such simple models. Namely, even if the behaviour rules of each individual (agent) are simple, the behavior of the swarm can be complicated. Reynolds called this kind of agent boid and tried to generate complicated swarm behavior by computer graphic (CG) animation [21]. Kennedy and Eberhart developed a PSO concept. PSO is basically developed through simulation of bird flocking in two-dimension space. The position of each agent is represented by $X Y$ axis position and the velocity is expressed by $v x$ (the velocity of axis) and $v y$ (the velocity of $Y$ axis). Modification of the agent position is realized by the position and velocity information.

Bird flocking optimizes a certain objective function. Each agent knows its best value so far (pbest) and its $X Y$ position. The velocity of each agent can be modified by the following equation:

$v_{i}^{k+1}=w v_{i}^{k}+c 1$ rand $1^{\times}\left(\right.$pbest $\left._{i}-s_{i}^{k}\right)+c 2$ rand $2^{\times}\left(\right.$gbest $\left._{i}-s_{i}^{k}\right)$

Where:

$v_{i}^{k} \quad$ velocity of agent at iteration;

$w \quad$ weighting function;

$c_{j} \quad$ weighting factor;

rand random number between 0 and 1 ;

$s_{i}^{k} \quad$ current position of agent at iteration ;

pbest $_{i} \quad$ pbest of agent ;

gbest $_{i}$ gbest of the group.

The right-hand side (RHS) of (3) consists of three terms (vectors) like three vectors of boid. The first term is the previous velocity of the agent. The second and third terms are used to change the velocity of the agent. 


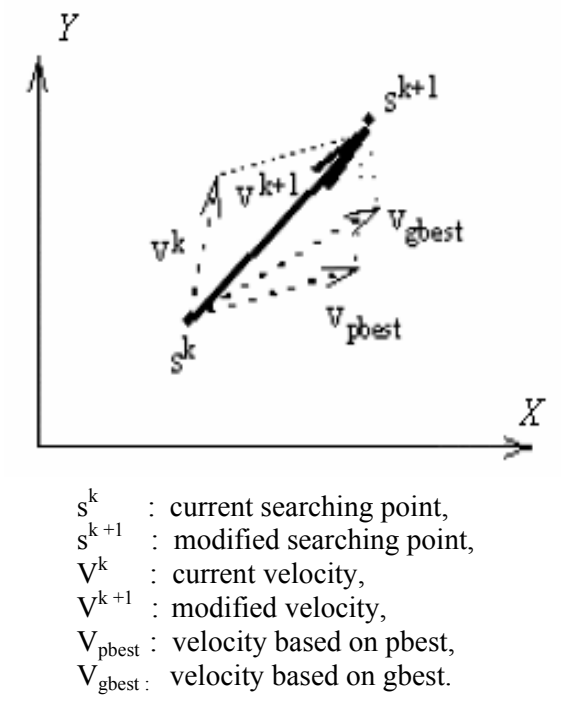

Fig. 4. Concept of modification of a searching point by PSO.

The current position (searching point in the solution space) can be modified by the following equation:

$$
S_{i}^{k+1}=S_{i}^{k}+v_{i}^{k+1}
$$

Fig. 4 shows a concept of modification of a searching point by PSO. The PSO algorithm can be expressed as follows (see Fig. 5):

1. State Variables (Searching Point): State variables (states and their velocities) can be expressed as vectors of continuous numbers. PSO uses multiple searching points for search procedures.

2. Generation of Initial Searching Points (Step 1 in Fig. 5): Initial conditions of searching points are usually generated randomly within their allowable ranges.

3. Evaluation of Searching Points (Step 2 in Fig. 5): The current searching points are evaluated by using the objective functions of the target problem. Pbests and gbest can be modified by comparing the evaluation values of the current searching points, and pbests and gbest.

4. Modification of Searching Points (Step 5 in Fig. 5): The current searching points are modified using the state equations (23) and (24).

5. Stop Criterion (Step 4 in Fig. 5): The search procedure can be stopped when the current iteration number reaches the predetermined maximum iteration number. The last gbest can be output as a solution.

\section{OBJECTIVE FUNCTION FORMULATION}

A power system is considered to be well damped if the damping for all eigenvalues is greater than 5\% [24].

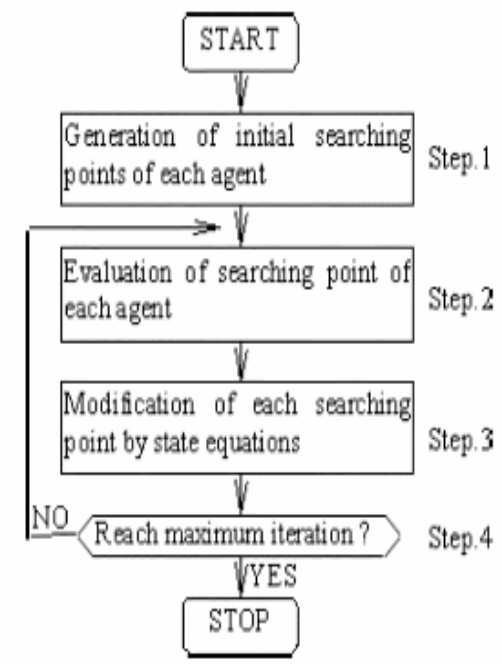

Fig. 5. General flowchart of PSO.

Therefore, the goal of AG and PSO based optimization procedure is to achieve $5 \%$ or greater of damping for all modes by exploring the search space of admissible controllers parameters.

As mentioned before (section II), the damping controller used in this paper is of fixed structure type given by:

$$
K_{i}(s)=k_{i}\left(\frac{s T_{\omega i}}{1+s T_{\omega i}}\right)\left(\frac{K_{r i}}{1+s T_{r i}}\right)^{2} \quad, i=1, . ., l .
$$

Where $l$ is the number of the controller.

From the viewpoint of a washout function, the precise value of the associated time constant $T_{w i}$ is not critical. The main consideration is that it should be small enough such that stabilizing signals at the frequencies of interest will be relatively unaffected [25]. The $k_{i}$ gain is fixed and considered equal to the unit. For these reasons it is considered to be known parameter. As a result we left up with $K_{r i}, T_{r i}$ and $G_{I i}$ (defined in section II) to be the parameters that should be determined by the tuning procedures. Let $\lambda_{j}=\alpha_{j} \pm \beta_{j}$ be the $\mathrm{j}$-th eigenvalue (mode) of the closed loop system. Then, the damping coefficient $\xi_{j}$ of the $j$-th eigenvalue is defined with the following equation:

$$
\xi_{j}=-\frac{\alpha_{j}}{\sqrt{\alpha_{j}^{2}+\beta_{j}^{2}}}
$$

Let $\Gamma_{r}$ be a vector of damping coefficients $\xi_{\mathrm{j}}, j=1, \ldots, n$ for the $r$-th operating condition. Where $n$ is the total number of modes of the closed loop system. Then, the optimization problem to be solved by the AG and PSO can be written in the following from:

$$
\max F=\left(\min \left(\min \Gamma_{r}\right)\right) \quad r=1, \ldots, m .
$$


Subject to :

$$
\begin{aligned}
& K_{r i \min } \leq K_{r i} \leq K_{r i \max } \\
& T_{r i \min } \leq T_{r i} \leq T_{r i \max } \\
& G_{I i \min } \leq G_{I i} \leq G_{I i \max }
\end{aligned}
$$

Where $m$ is the total number of operating conditions under consideration.

This problem is generally very hard to solve by trail and error or whit traditional techniques since continuity of the objective function cannot be established. In the next section Genetic Algorithm and Particle Swarm Optimization techniques will be used to solve this problem.

\section{RESULTS AND DISCUSSION}

The proposed tuning procedure is then applied to a 2area 4-machine system [10], depicted in figure 6.

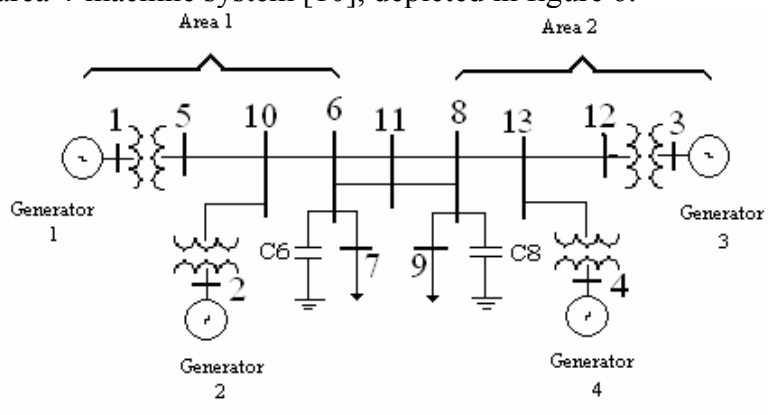

Fig. 6. One-Line Diagram of the 2-area 4-machine.

In this study, tuning of a two input SVC damping controller installed on the Generator G2 and G4 is investigated. The sites of SVCs are defined by the contribution factors matrix [15], [27]. Table 1 gives the generators contribution factors for the electromechanic modes, after appling small disturbance equal to $5 \%$ of the value of the electromechanical torque, for the uncontrolled system. The results indicate that machines G2 and G4 are the optimum locations for installing SVCs to damp out the local and interarea modes of oscillations.

Table1. Generators contribution factors

\begin{tabular}{|c|c|c|c|c|}
\hline Modes & G1 & G2 & G3 & G4 \\
\hline$-0.0322 \pm 6.4630 \mathrm{i}$ & 0.1593 & $\mathbf{0 . 5 5 7 9}$ & 0.0189 & $\mathbf{0 . 2 6 3 5}$ \\
$-0.0322 \pm 4.0327 \mathrm{i}$ & $\mathbf{0 . 7 5 0 5}$ & $\mathbf{0 . 2 4 3 6}$ & 0.0052 & 0.0006 \\
$-0.0322 \pm 5.2166 \mathrm{i}$ & 0.0005 & 0.0210 & $\mathbf{0 . 7 6 3 6}$ & $\mathbf{0 . 2 1 4 6}$ \\
\hline
\end{tabular}

The inductive susceptance $B$ of SVC is given equal to 0.6 p. u. The parameters of Genetic Algorithm and Particle Swarm Optimization techniques are given in tables 2 and 3 respectively. In the objective to increase the search space, the following operating conditions are considered:
Table. 2. GA parameters

\begin{tabular}{|l|c|c|}
\hline AG parameters & & Search space \\
\cline { 1 - 2 } encoding & Binary & \multirow{2}{*}{$0.1 \leq K_{\mathrm{r}} \leq 100$} \\
\cline { 1 - 2 } Population size & 40 & \multirow{2}{*}{$0.1 \leq G_{I i} \leq 10$} \\
\cline { 1 - 2 } Chromosome size & 96 & $0.01 \leq T_{\mathrm{r}} \leq 1$ \\
\cline { 1 - 2 } crossover rates $\left(\mathrm{p}_{\mathrm{c}}\right)$ & 0.1 & \\
\hline Points of crossover & 2 & \\
\hline mutation rates & 30 & \\
\cline { 1 - 2 } Number of iteration & &
\end{tabular}

Table. 3. PSO parameters

\begin{tabular}{|l|c|c|}
\hline PSO parameters & & Search space \\
\hline Population size & 10 & \\
\cline { 1 - 2 } Number of iteration & 10 & \multirow{2}{*}{$0.1 \leq K_{\mathrm{r}} \leq 100$} \\
$\left(\right.$ iter $\left._{\max }\right)$ & & $0.1 \leq G_{I i} \leq 10$ \\
\cline { 1 - 2 }$\omega_{\max }$ & 0.9 & $0.01 \leq T_{\mathrm{r}} \leq 1$ \\
$\omega_{\min }$ & 0.8 & \\
\hline $\mathrm{c}_{1}$ & 2.4 & \\
$\mathrm{c}_{2}$ & 2.4 & \\
\hline
\end{tabular}

- $\quad$ Loss of the line (6-11) and (8-11).

- High load, equal to 1.8 of the nominal load.

- Weak load, equal to 0.3 of the nominal load.

- Appling small disturbance equal to $5 \%$ of the value of the electromechanical torque from the generator G2, for nominal load.

Table 4 shows the obtained SVCs parameters by GA and PSO optimization. It also, presents the parameters of SVCs without optimization.

Table. 4. SVC parameters.

\begin{tabular}{|c|c|c|c|c|c|c|}
\hline & \multicolumn{2}{|c|}{$\begin{array}{c}\text { Without } \\
\text { Optimization }\end{array}$} & \multicolumn{2}{|c|}{$\mathrm{GA}$} & \multicolumn{2}{c|}{ PSO } \\
\hline $\begin{array}{c}\text { SVC } \\
\text { parameters }\end{array}$ & $\mathrm{SVC}_{1}$ & $\mathrm{SVC}_{2}$ & $\mathrm{SVC}_{1}$ & $\mathrm{SVC}_{2}$ & $\mathrm{SVC}_{1}$ & $\mathrm{SVC}_{2}$ \\
\hline $\begin{array}{c}\text { Population } \\
\text { size }\end{array}$ & \multicolumn{2}{|c|}{-} & \multicolumn{2}{|c|}{40} & \multicolumn{2}{c|}{10} \\
\hline $\begin{array}{c}\text { Number of } \\
\text { iteration }\end{array}$ & \multicolumn{2}{|c|}{-} & \multicolumn{2}{|c|}{30} & \multicolumn{2}{c|}{10} \\
\hline$K_{r i}$ & 5.1 & 0.1 & 85.6206 & 90.4620 & 55.7862 & 64.7000 \\
\hline$T_{r i}$ & 0.8 & 0.5 & 0.4581 & 0.0693 & 0.0543 & 0.2975 \\
\hline$G_{I i}$ & 10 & 10 & 2.0481 & 4.1414 & 3.6836 & 6.6613 \\
\hline
\end{tabular}

The robustness of the GA and PSO designed controllers is validated for three operating conditions representing different scenarios as follows:

- $\quad$ Loss of the line (6-11).

- High load, equal to 1.5 of the nominal load.

- Weak load, equal to 0.5 of the nominal load.

The simulation results for all three operating conditions are shown in Figs. 7 to 9. 

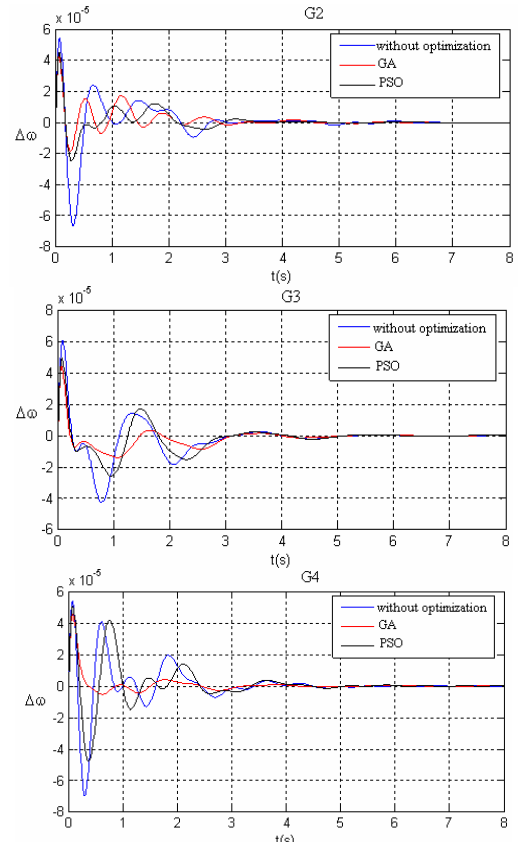

Fig. 7. Speed Deviations for high load condition.
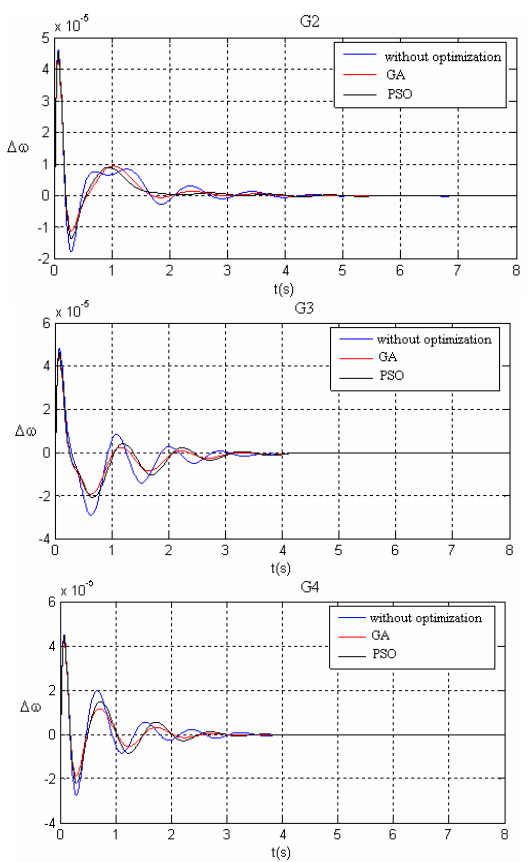

Fig. 8. Speed Deviations for weak load condition.

We can notice that the damping is improved by the PSO optimisation but it more effectively damped by the GA. Figs. 10 to 12 show a comparison plot between the eignenvalues of the cases without and with optimization for the operating conditions. It can be seen clearly that the system damping with the designed controllers has been improved to more than 5\%. For the high load condition and the line outage, the GA method is more effective than the
PSO. When the load is weak, the system damping improved by PSO method is more effective than GA-based technique.
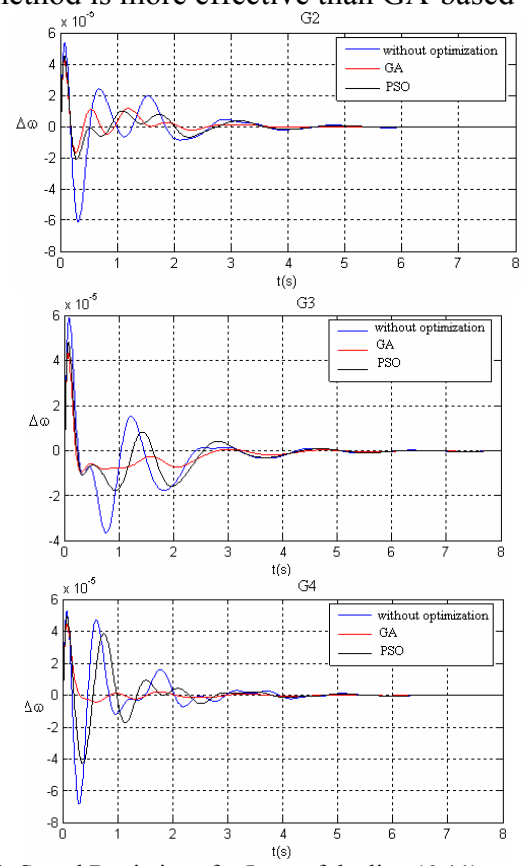

Fig. 9. Speed Deviations for Loss of the line (6-11) condition.

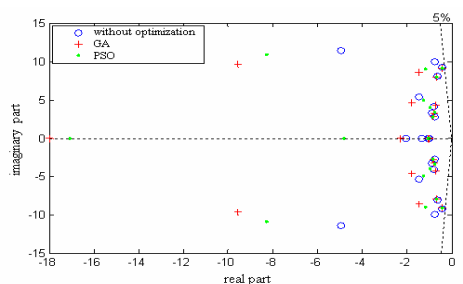

Fig.10. Eigenvalues of the system with and without optimization methods (GA and PSO) for the high load condition.

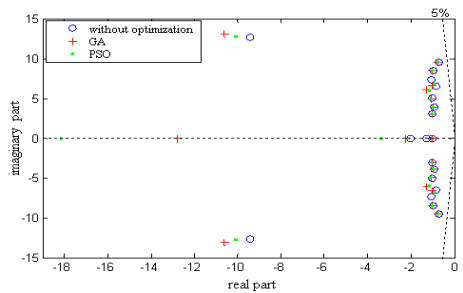

Fig.11. Eigenvalues of the system with and without optimization methods (GA and PSO) for the weak load condition.

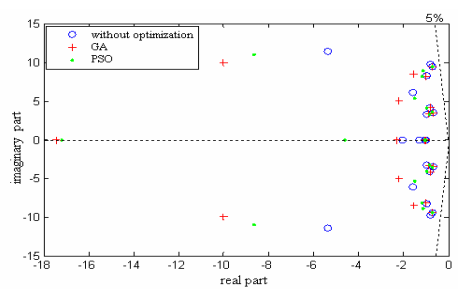

Fig.12. Eigenvalues of the system with and without optimization methods (AG and PSO) for the line outage condition. 


\section{CONCLUSION}

In this paper, a robust power system damping controller design method using Genetic Algorithm and Particle Swarm Optimization was presented. The method was successfully applied to design an SVC damping controller in a 2-area 4-machine system. Using an appropriate set of synthesized controller parameters, the designed controllers enhance the system performance and a stabilized system under abnormal conditions is obtained.

Both GA and PSO have proven to be very effective but PSO has many advantages over GA:

- Faster Convergence.

- Smaller population size.

- Lower number of iterations.

\section{REFERENCES}

[1] Lin, C.E., Chen, T.C., and Huang, C.L.: 'Optimal control of a static Var compensator for minimization of line loss', Electr. Power Syst. Res., 1988, 15, pp. 5141

[2] O’brien, M., and Ledwich, G., ' Static reactive-power Compensator controls for improved system stability', IEE Proc. C, 1987,134 , pp. $38-42$

[3] Ramos, A.J.P., and Tyll, H.: 'Dynamic performance of a radial weak power system with multiple static Var compensator'. Paper 89 WM 183-5 PWRS, presented at the IEEE/PES 1989 Winter meeting New York.

[4] Gerin-Lajoie, L., Scom, G., Breault, S., Larsen, E.V., Baker, D.H., and Imece, A.F.: ' Hydro-Quebec multiple SVC Application control stability study', IEEE Trans., 1990, PWRD-S, pp. 1543-1551.

[5] Abdel-Madjid Y. L., Abido M. A., Al-Baiyat S. and Mantawy A. H., "Simultaneous Stabiliszation of multi-machine power system via genetic algorithms.", IEEE Transactions on Power Systems., vol. 14, pp. 1428-39, Nov. 1999.

[6] Farag W. A., Quintana V. H. and Lambert-Torres G., " Enhacing The transient stability of multi-machine power systems using Intelligent techniques. ", Bulk-Power-Systems-Dynamics-andControl-IV-Restructuring-Symposium-Proceedings, Nat. Tech. Univ. Athens. Athens, Greece, pp. 117-25,1998.

[7] Taranto G. N. and Falcão D.M., " Robust decentralised control design using genetic algorithms in power system damping control.", IEE Proc.-Gener. Transm. Distrib., Vol. 145, No. 1, January 1998.

[8] H. Yoshida, K. Kawata, Y. Fukuyama and Y. Nakanishi, "A particle swarm optimization for reactive power and voltage control considering voltage stability.", IEEE International Conference on Intelligent System Applications to Power Systems (ISAP'99), Rio de Janeiro, April 4-8, 1999.

[9] Al-Hinai. A, A. Feliachi. A, "Control of microturbines in a power distribution system using particle swarm optimization", West Virginia University, 2004.

[10] Hasanovié. A., A Simulation and Analysi Toolbox for Power Systems and Genetic Algorithm Based Damping Controller Design, Master thesis, Morgantown, West Virginia 2001.

[11] O'brien, M., and Ledwich, G., Static reactive power compensator controls for improved system stability, IEE Proc. C, 1987, 134, (1), pp. 3842.

[12] Cheng, C.H., and HSO, Y.Y.: Application of a power system Stabilizer and a static VAr controller to a multimachine power system, IEE Proc. C, 1990, 137, (1), pp. 8-12.

[13] Dash, P. K., Panda, D. C., Sharad, A.M., and Hill, E. F. : Adaptive controller for static reactive power compensators in power systems, IEE Proc. C, 1987, 134, (3), pp. 256-264.

[14] El-Emary. A. A, Static VAr compensator, Synchronising torque coefficient, IEE Proceedings online no. 19960544 , Paper first received $13^{\text {th }}$ Decmber 1994 and in revised form $12^{\text {th }}$ April1996.

[15] Yu Y. N., Electric power system dynamics, Academic Press Inc.,
India, 1983)

[16] Gibbard, M.J.: Co-ordination of multimachine stabilizer gain settings for a specified level of system damping performance, IEE Proc. C, 1982, 129, (2), pp. 4548

[17] D. E. Goldberg, Genetic Algorithms in Search, Optimization and Machine Learning, Addison-Wesley, Reading, MA, 1989.

[18] M. Srinivas and L. M. Patnaik, "Genetic Algorithms: A Survey, , Computer, Vol. 24, No. 6, pp. 17-26, 1994.

[19] J. Kennedy and R. Eberhart, "Particle swarm optimization," in Proc. IEEE Int. Conf. Neural Networks, vol. 4, Perth, Australia, 1995, pp. 1942-1948.

[20] Y. Fukuyama, et al., " A particle swarm optimization for reactive power and voltage control considering voltage security assessment," IEEE Trans. Powe r Syst., vol. 15, pp. 1232-1239, Nov. 2000.

[21] C. Reynolds, "Flocks, herds, and schools: A distributed behavioral model," Computer Graphics, vol. 21, no. 4, pp. 25-34, 1987.

[22] R. Boyd and P. Recharson, Culture and the Evolutionary Process. Chicago, IL: Univ. of Chicago Press, 1985.

[23] E. Bonabeau, M. Dorigo, and G. Theraulaz, Swarm Intelligence: From Natural to Artificial Systems. London, U. K. : Oxford Univ. Press, 1999.

[24] , Swarm Intelligence. San Mateo, CA: Morgan Kaufmann, 2001.

[25] Graham. R, Power System Oscillation, M. A. Pai, Ed., Norwell: Kluwer Academic Publishers, 2000.

[26] Antonio L. B. do Bomfim, Glauco N. Taronto, Member, IEEE and Dialma M. Falcao, Senior member IEEE. "Simultaneous Tuning of Power System Damping Controllers using Genetic Algorithms." IEEE Transactions on Power Systems, Vol. 15, No. 1, February 2000 .

[27] Singhvi. V., Small Signal Stability of an Unregulated Power System, Master's Thesis, Department of Electrical and Computer Engineering, Mississippi, December 2002 